\title{
Does a patient's personality style impact health outcomes?
}

Kam A Mclntosh

This case describes a young man with severe haemophilia B who uses the "avoiding" mode of behaviour, leading to isolation and reduced ambulation. Health care providers promoting health change behaviour were able to empower the patient to direct his care toward his desired outcomes. As his self-awareness increases, he begins to use another mode of behaviour: compromising. ThomasKilmann's conflict management framework is a novel approach to bringing two sides together in a working partnership. This challenging case shows the balance between a patient's self-care and symbiotic interaction with HCPs to achieve successful health outcomes.

Keywords: haemophilia B; arthropathy; conflict; personality; behaviour modes

Health problems arise despite the best efforts of the patient and health care providers, but personality may influence the outcome. Self-awareness and self-management may enhance conflict management when variance and conflict mean a state or condition marked by a lack of agreement or harmony [1]. Five modes of behaviour competing, collaborating, compromising, avoiding and accommodating - are frequently used in difficult health situations [2,3]. Overuse of one of these behaviour modes may lead to resolution failure, dissatisfaction and poor health outcomes $[4,5]$.

There are many models that can be used to resolve challenges. Some experts feel personality style may be more important than the problem itself [5]. The ThomasKilmann conflict model defines the five modes of behaviour to explore incongruent opinions:

- Competing - "If I'm involved in conflict, I want to be in charge"

- Compromising - "Let's look for middle ground; if everyone gives in a little, we'll have a speedy settled solution"

- Collaborating - "Let's take the time to put our heads together find a solution everyone likes"

- Avoiding - conflict is ignored or stalled, "I don't want to be involved"

- Accommodating - "I'll just give in, it'll save further problems" $[3,6]$.

Kam Mclntosh, Patient/Nurse Educator, British Columbia Provincial Bleeding Disorders Program - Adult Division, Burrard Building, Room 491 St. Paul's Hospital, 1081 Burrard Street, Vancouver, BC, Canada V6Z 1 Y6. E-mail: kmcintoshaprovidencehealth.bc.ca

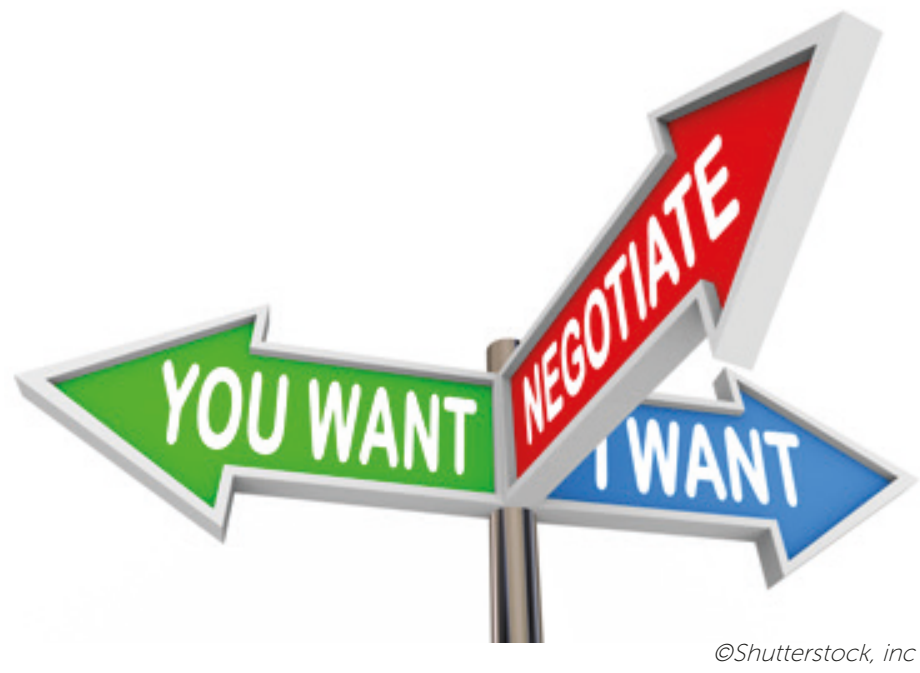

The degree of assertiveness and cooperativeness further influences the five personality style modes: my way, halfway, our way, no way and your way [7].

Patients with severe haemophilia B, an inherited bleeding disorder, lack a protein necessary to clot blood. Beginning in infancy, the patient's health care provider and parents begin sharing the responsibility of managing this rare bleeding disorder. Joint bleeds cause patients to suffer acute pain and subsequent arthropathy [8]. Prophylactic factor IX treatment has changed the goal of haemophilia programs from an emphasis on treating life-threatening bleeds to a focus on managing chronic disease, including interventions to reduce the risk of long-term disability [9].

Case history

At the age of 19 years old, a Canadian man with haemophilia $B$ was advised by an orthopaedic surgeon that he had the ankles of an 80-year-old man and ankle fusion would not be a possibility for seven years. Now, at 23 years of age, he has chronic haemophilic arthropathy in his right and left ankles. He is in receipt of disability benefits and lives with a roommate while finishing grade 12 high school. He plans to continue his education in social sciences.

Historically, after a bleed, he avoided using the clinic's protocols of administering 1-2 follow-up factor IX doses and contacting the clinic when additional follow-up treatment doses were required. His fear of having further bleeds in his ankles caused him to self-prescribe follow-up factor IX treatments for 5-13 days. Despite advice and education from his health care providers, and being encouraged to 
telephone the clinic should he require additional followup treatments, the patient continued to over treat. The provision of information alone had little or no impact.

The patient's behaviour began to change after he was asked to describe his prescribed treatment plan. Although not following the plan; he described it fully and accurately. On being asked about a treatment plan he envisioned would decrease bleeds and follow-up treatments, the young man compromised his wish to treat with recombinant factor IX 1500 units daily, and devised his own unique treatment plan of 1000 units daily (HCP plan) for 6 days with one weekend day of 3000 units (patient plan). The patient's self-directed plan, approved by clinic physicians, went on to sustain a year of zero bleeds.

In May 2015, the patient suffered a bleed from accidental trauma to his left ankle. Despite prophylaxis, the arthropathic ankle bled again in October as a result of physical overuse. Because the pain did not resolve to weight bearing within 1-2 weeks, he limited his activities to home and used crutches or hopped about. By January 2016, significant muscle wasting had occurred in his left leg, including the beginning of a contracture in the left foot. He avoided discussing the severity of the situation when a factor order was placed. As he did not trust the effectiveness of his factor IX, he overused to the extent of 53 follow-up doses after the two left ankle bleeds. His belief that frequent and high doses of factor are the best treatment for bleeds causing pain for longer than 1-2 weeks was influenced by having been admitted to hospital for a month for a psoas bleed at 16 years old and receiving high and frequent doses of factor. He has deferred completing Grade 12 until his ankles are "better".

\section{Management and outcomes}

Frequent follow-up appointments with the physiotherapist were arranged. Even though the patient had a trough of $5 \%$ on 1000 units daily, he still had breakthrough bleeds in 2015 , thought to be attributed to (a) cycle of unresolved synovitis/inflammation and/or (b) possibly lower factor

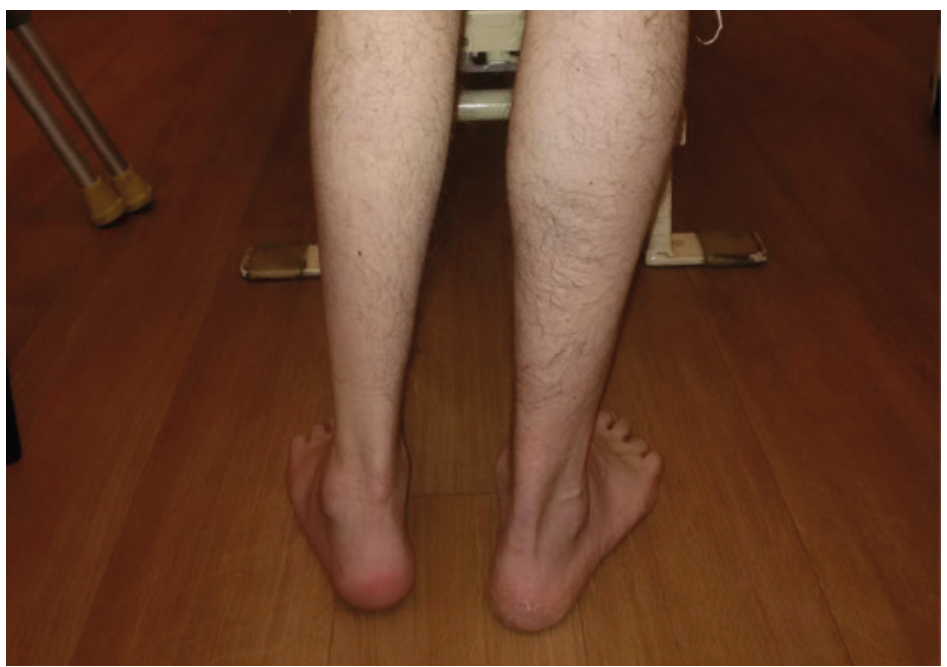

Figure 1: In January 2016, the patient avoided weight-bearing
IX penetration/distribution in the ankle. He was therefore switched to a trial of plasma-derived factor IX in January 2016 to see if it was more effective, and prescribed celecoxib $100 \mathrm{mg} \mathrm{BID}$ to reduce the inflammation and prevent rebleeding. At this stage, the patient avoided weight-bearing (Figure 1). He was also on the waiting list for a possible synovectomy.

The patient explained that the physiotherapy strengthening exercises he was using to rebuild the muscles caused inflammation, but the celecoxib cooled the inflammation, preventing bleeds. This combination reverted muscle wasting to muscle building. In addition, the patient reported that he felt his ankle braces (introduced January to April 2016) provided support. He said: "I would use the braces for about 8 - 10 hours a day on the physio's recommendation. She mentioned that even though primarily those braces are used for support, which is what I use them for on a daily basis, that it would also prevent my ankle from healing in a slouched position." To date, plasma-derived factor IX has prevented further bleeds.

\section{Discussion}

Actively engaging the patient in his care has achieved the best results. The patient describes his awareness of his pain, activity and joint range of motion as helping him to selfmanage his bleed rate. Using the experience of the patient and the expertise of the HCPs to actively support patient autonomy in all aspects of decisions related to haemophilia management, the British Columbia Hemophilia Adult Team approach de-emphasises "adherence" as the primary goal, and focuses on a prophylaxis plan that is customised by the patient and aligned with his priorities [10]. Gaining the patient's insight and opinion promotes health change behaviour, empowering him to direct his care toward his desired outcomes.

When health challenges arose, our patient described his two personality styles as Avoiding and Compromising. Neither personality style is right or wrong, but it is helpful to detect the patient's preferred personality style(s) and for the

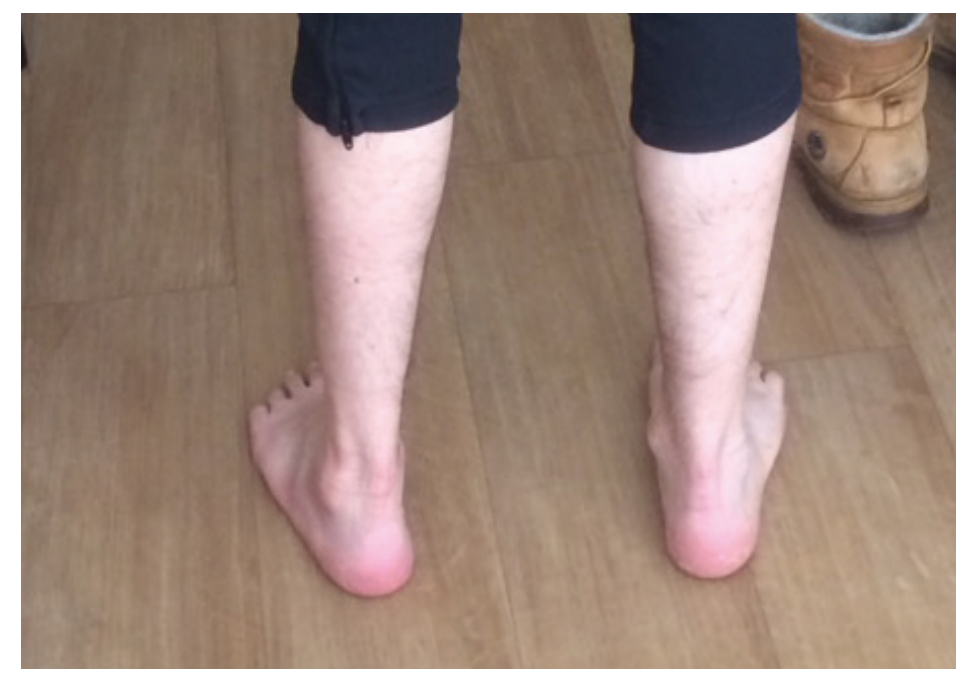

Figure 2: By April 2016, he was able to walk without crutches 
health care providers to have insight into their personality behaviour mode(s) [11]. If the health care provider tends to be a collaborator and wants to discuss the problem at length with the patient, but the patient wants to use compromise to find middle ground or to simply avoid it, a collaborative approach could impair resolution [12]. Avoiding an issue may seem effective in the short-term, but is not a longterm solution. If overuse of avoidance gradually weakened the left ankle, then the overuse of a particular personality style may weaken efforts to manage the problem.

Each of the five personality styles can be effective in the right circumstances; the key is not to overuse one or two preferred styles indiscriminately. Using all five styles appropriately, depending on the situation, enables a more balanced approach. Initially, the patient avoided weightbearing for four months. However, he compromised, creating common ground with the health care providers, he was empowered to direct his care toward his desired outcomes. The patient reported that he was willing to follow the health care provider's treatment plan for rehabilitation, but wanted control over the timeline to become weightbearing without crutches (Figure 2).

\section{Conclusion}

It is important that haemophilia health care providers have awareness of multiple strategies to bring down barriers blocking optimum haemophilia health outcomes in patients who practice self-care. An awareness of personality styles may bring two sides together and promote a good working relationship to achieve optimum health outcomes.

\section{Next steps}

HCPs may have the skills to discern if a patient is competing, collaborating, compromising, avoiding or accommodating. However, are they aware of their own personality styles? Soft skills enhance collaboration and partnership between health care providers and others [11]. We plan to examine this further by inviting 120 haemophilia nurses to take part in a quality improvement research project. Some 30 Canadian nurses at their AGM and 80-100 from around the world at the WFH Congress in Orlando Florida in July 24, 2016 will be asked to complete a TKI questionnaire (ThomasKilmann Conflict Mode Instrument). This is a tool that will enable them to enhance their conflict management skills through self-awareness, by determining their most and least frequent behaviour modes in work relationships. The $\mathrm{TKI}$ questionnaire is a leading measure of conflict-handling modes that has been used in research for more than 40 years. This instrument has been used over four million times and has been featured in hundreds of research studies. The TKI CPP Inc website notes that the instrument is constantly reviewed and updated for differences on median TKI scores between men and women, as well as between ethnic groups, organizational levels, educational levels and are negligible in terms of practical importance. as posing a conflict or bias.

This is an Open Access article distributed under the terms of the Creative Commons Attribution License (http:// creativecommons.org/licenses/by/2.0), which permits unrestricted use, distribution, and reproduction in any medium, provided the original work is properly cited.

\section{Acknowledgments}

The author wishes to thank Dr Shannon Jackson and Dr Linda Sun for their assistance.

\section{References}

1. Merriam-Webster Dictionary. Conflict. Available from: http://www. merriam-webster.com/dictionary/conflict (accessed 15 April 2016).

2. Ogunyemi D, Tangchitnob E, Mahler Y, et al. Conflict styles in a cohort of graduate medical education administrators, residents, and boardcertified physicians. J Grad Med Educ 2011;Jun;3(2):176-81. doi:10.4300/ JGME-D-10-00184.1.

3. Kilmann RH, Thomas KW. Developing a forced-choice measure of conflict

management behavior: the MODE instrument. Educ Psychol Meas 1977;37(2):309-25.

4. Losa Iglesias ME, Becerro de Bengoa Vallejo R. Conflict resolution styles in the nursing profession. Contemp Nurse 2012;43:73-80.

5. Kilmann R. Conflict management and change management case study with the TKI tool [video]. 2014. Available from: http://youtu.be/ Tnf1OKoGSys (accessed 21 April 2016).

6. Whitworth BS. (2008), Is there a relationship between personality type and preferred conflict-handling styles? An exploratory study of registered nurses in southern Mississippi. J Nurs Manag 2008;16:921-32. doi:10.1111/ j.1365-2834.2008.00918.x.

7. Towler J. Managing conflicts effectively: workplace conflict management and how to do it well. Available from: http://creativeorgdesign.com/article. $\mathrm{htm}$ ? id=1128title=Managing_Conflicts_Effectively (accessed 9 April 2016). 8. Rodriguez-Merchan EC, Jimenez-Yuste V, Aznar JA, et al. Joint protection in haemophilia. Haemophilia 2011;17 Suppl 2:1-23. doi:10.1111/ j.1365-2516.2011.02615.x.

9. Skinner MW. WFH: closing the global gap -- achieving optimal care. Haemophilia 2012;18 Suppl 4:1-12. doi:10.1111/j.1365-2516.2012.02822.x. 10. Gue $D$, Squire $S$, Mclntosh $K$, et al. Joining the patient on the path to customized prophylaxis one hemophilia team explores the tools of engagement. J Multidiscip Healthc 2015; 8:527-34.

11. Guerin TT. Relationships matter: the role for social-emotional learning in an interprofessional global health education. J Law Med Ethics 2014;42 Suppl 2:38-44. doi:10.1111/jlme.12186

12. CPP Inc. Four tips for managing conflict [video]. 2014. Available from: https://www.youtube.com/watch?v=QJiJ95mHftE (accessed 16 April 2016).

13. Zweibel EB, Goldstein R, Manwaring JA, Marks MB. What sticks: how medical residents and academic health care faculty transfer conflict resolution training from the workshop to the workplace. Conflict Resolution Quarterly 2008:25:321-50. doi:10.1002/crq.211.

14. Trippe B, Baumoel D. Beyond the Thomas-Kilmann model: into extreme conflict. Negotiation Journal 2015;31:89-103. doi:10.1111/nejo.12084.

15. Riasi A. The relationship between principals' reward power and their conflict management styles based on Thomas-Kilmann conflict mode instrument. Management Science Letters 2015;5(6):611-8. doi:10.5267/j. msl.2015.4.004.

16. Ephrom L. (Omineca Transition House support worker). Working together successfully - managing differences [personal communication]. 11 October

\section{Disclosures}

The author has advised no interests that might be perceived 